\title{
EVALUACIÓN DE LA SUPLEMENTACIÓN CON SELENIO ORGÁNICO Y SU EFECTO SOBRE EL DESEMPEÑO PRODUCTIVO Y REPRODUCTIVO DE VACAS LECHERAS EN PASTOREO EN COSTA RICA ${ }^{1}$
}

\author{
Jeffry Sánchez-Salas ${ }^{* *}$, Jorge Alberto Elizondo Salazar ${ }^{2 *}$, Evelio Víquez Matei $^{* *}$, Carlos Orozco- \\ Vidaorreta $^{* * *}$
}

Palabras clave: Minerales traza, leche, leche enriquecida, nutrición, ganado de leche. Keywords: Trace minerals, milk, enriched milk, nutrition, dairy cattle.

Recibido: $26 / 04 / 13$

\section{RESUMEN}

El objetivo fue evaluar el efecto de la suplementación con levadura selenizada, derivada de una cepa específica de Saccharomyces cerevisiae (CNCM I-3060), sobre el comportamiento productivo y reproductivo y según las concentraciones de selenio en la leche de vacas lecheras en pastoreo. Vacas Holstein multíparas, $(\mathrm{n}=40)$ con un peso promedio de $607 \pm 62 \mathrm{~kg}$ y una

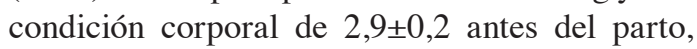
fueron asignadas al azar a 1 de 2 tratamientos. El tratamiento control consistió de una dieta basal que suministró 0,7 mg de Se. $\mathrm{kg}^{-1} \mathrm{MS}$. El tratamiento experimental consistió de la misma dieta basal suplementada con 3,0 mg de Se, del día 5 al 56 de lactancia. La producción y composición de la leche, el CCS y el contenido de Se en la leche se determinaron a los 5, 14, 28, 42 y 56 días de lactancia. También se tomaron muestras de sangre de cada animal durante los mismos días, para medir la glutatión peroxidasa (GSH-Px) y la concentración de Se. Para evaluar el desempeño reproductivo, los ovarios fueron examinados por ultrasonografía transrectal en los días 22 y 57 posparto. Días abiertos, días a primer servicio y servicios por concepción también fueron

1 Proyecto 737-A9-247. Inscrito en la Vicerrectoría de Investigación. Universidad de Costa Rica. Costa Rica.

2 Autor para correspondencia. Correo electrónico: jorge.elizondosalazar@ucr.ac.cr
Aceptado: 20/08/13

\begin{abstract}
Supplementation with organic selenium and its effect on productive and reproductive performance in grazing dairy cows in Costa Rica. The objective was to evaluate the effect of supplementation with selenized yeast, derived from a specific strain of Saccharomyces cerevisiae (CNCM I-3060), on productive and reproductive performance and on selenium concentrations in milk of grazing dairy cows. Multiparous Holstein cows $(n=40)$, with an average body weight of $607 \pm 62 \mathrm{~kg}$ and a body condition score of $2.9 \pm 0.2$ before parturition, were randomly assigned to 1 of 2 treatments. Control treatment consisted of a basal diet supplying $0.7 \mathrm{mg}$ of Se. $\mathrm{kg}^{-1}$ dry matter. Experimental treatment consisted of the same basal diet supplemented with $3.0 \mathrm{mg}$ of Se, from day 5 to 56 of lactation. Milk production, milk composition, SCC, and Se content of milk were determined at day 5, 14, 28,42 , and 56 of lactation. Blood samples from each cow were also taken during the same days, to measure glutathione peroxidase (GSH-Px) and Se concentration. To evaluate reproductive performance, the ovaries were examined by transrectal ultrasonography at days 22 and 57

\footnotetext{
* Estación Experimental Alfredo Volio Mata, Facultad de Ciencias Agroalimentarias. Universidad de Costa Rica. Cartago, Costa Rica.

** Cooperativa de Productores de Leche Dos Pinos R.L. Costa Rica.

*** Alltech, Inc. Costa Rica.
} 
registrados. La producción y composición de la leche y el CCS no difirieron entre tratamientos durante el experimento. La suplementación con selenio no alteró la concentración de GSH-Px (199,0 vs. $\left.220,3{\mathrm{U} . \mathrm{g}^{-1}}^{\mathrm{de}} \mathrm{Hb}\right)$ ni la concentración de Se en sangre (166,4 vs. 184,2 $\left.\mu \mathrm{g} \cdot \mathrm{l}^{-1}\right)$. No obstante, la suplementación con selenio orgánico aumentó $(\mathrm{p}<0,01)$ la eficiencia aparente de transferencia de Se a la leche (7,9 vs. $9,9 \%$ ) y el contenido de Se (12,7 vs. 20,5 $\mu \mathrm{g} . \mathrm{l}^{-1}$ ), por lo que la leche derivada de vacas suplementadas con levadura de Selenio podría ser una vía útil para contribuir a la ingesta diaria de Se en humanos, que permitieran alcanzar las recomendaciones dietéticas diarias que contribuyan como alimento funcional a solventar problemas relacionados con la deficiencia de este nutriente.

\section{INTRODUCCIÓN}

El selenio (Se) es considerado uno de los elementos trazas más controversiales, pues a pesar de ser tóxico en dosis elevadas, su deficiencia se ha convertido en un problema global debido a su esencialidad para un adecuado funcionamiento del organismo, ya que es un componente estructural de la enzima Glutatión Peroxidasa (GSH-Px) y de otras selenoproteínas involucradas en la protección antioxidante (Lyons et ál. 2007).

En los últimos años, se ha demostrado que los suelos generalmente tienen una baja concentración de Se, que proveen a los forrajes y otros cultivos que crecen en ellos, cantidades inadecuadas del mineral que a su vez generan un incremento en la susceptibilidad a enfermedades y una disminución del desempeño productivo y reproductivo de los animales (Ceballos et ál. 2009).

Dicho aspecto ha ocasionado que el nivel de este elemento en los alimentos de origen animal para el consumo humano varíe entre regiones. Por ejemplo, en el caso de Costa Rica la Comisión Intersectorial de Guías Alimentarias ha considerado al Se como un nutriente emergente por su relación con la prevención de enfermedades postpartum. Days open, days to first service and services per conception were also recorded. Milk production, milk composition, and SCC did not differ between treatments during the trial. Selenium supplementation did not alter GSH-Px concentration (199.0 vs. $220.3 \mathrm{U.g}^{-1} \mathrm{Hb}$ ) or Se concentration in blood (166.4 vs. $184.2 \mu \mathrm{g} . \mathrm{l}^{-1}$ ). However, organic selenium supplementation increased $(\mathrm{p}<0.01)$ Se apparent efficiency of transfer into milk (7.9 vs. $9.9 \%$ ) and Se content of milk (12.7 vs. $\left.20.5 \mu \mathrm{g} .1^{-1}\right)$; therefore, milk derived from cows supplemented with selenized yeast could be a useful way to contribute to daily intake of Se in humans, allowing to reach the daily dietary recommendations and helping as a functional food to solve problems related to Se deficiency.

crónicas (CIGA 2007), si se considera que un $35 \%$ de la población adulta costarricense presenta deficiencias de Se, como lo evidencia la última Encuesta Nacional de Nutrición (Ministerio de Salud 2010).

En este sentido, una posible deficiencia de Se implicaría una limitada expresión de las selenoproteínas relacionadas con la con la habilidad protectora contra el daño oxidativo; sin embargo, es importante notar que la suplementación de Se en humanos y animales domésticos deficientes en Se, restaura la expresión de dichas selenoproteínas. Ante esto, una práctica común consiste en la suplementación con Se en las dietas de rumiantes, pues los ingredientes no contienen concentraciones adecuadas para alcanzar la alta demanda del mineral (Himeno e Imura 2000, Lyons et ál. 2007).

La práctica más común consiste en la suplementación con Se en su forma inorgánica, no obstante, el reemplazo de dicha fuente por Se orgánico ha sido probado como un medio efectivo de solventar los problemas de Se en el ganado lechero. Algunos trabajos que han comparado estos aportes, fueron recopilados por Weiss (2005), quien registró un incremento 
promedio del $16 \%$ en la actividad de la GSH-Px y del $20 \%$ en la concentración de Se en sangre. A su vez, esto resulta en una alternativa potencialmente efectiva para mejorar el nivel de consumo del Se en la población humana a través de leche enriquecida con este mineral, puesto que la suplementación de vacas lecheras con Se orgánico ha incrementado considerablemente la concentración de Se en este alimento (Givens et ál. 2004, Heard et ál. 2007, Doyle et ál. 2011).

Por esta razón, el objetivo de este estudio fue evaluar el efecto de la suplementación con selenio orgánico sobre la producción y composición láctea, contenido de selenio en leche y actividad de la Glutatión peroxidasa de vacas lecheras en pastoreo.

\section{MATERIALES Y MÉTODOS}

\section{Diseño experimental y manejo}

El experimento fue conducido en Poás, Alajuela, Costa Rica $\left(10^{\circ} 16^{\prime} \mathrm{N}, 84^{\circ} 19^{\prime} \mathrm{O}\right)$ entre Diciembre 2011 y Mayo 2012. El estudio involucró 40 vacas Holstein multíparas al momento del parto con un peso y condición corporal promedio de $607 \pm 62 \mathrm{~kg}$ y $2,90 \pm 0,21$, respectivamente; las cuales fueron asignadas aleatoriamente a 2 tratamientos. En ambos casos, las vacas pastorearon Kikuyo (Kikuyochloa clandestinum) con una disponibilidad de $27 \mathrm{~kg} . \mathrm{vaca}^{-1}$.día $\mathrm{a}^{-1} \mathrm{MS}$.

Las vacas fueron ordeñadas diariamente a las 0400 y 1500 h. Previo al ordeño, los animales consumieron una ración total mezclada (RTM) que consistió de $9 \mathrm{~kg}$ de silopaca de Kikuyo, $9 \mathrm{~kg}$ de subproductos de cervecería, $4 \mathrm{~kg}$ de alimento balanceado, $2 \mathrm{~kg}$ de melaza, $0,13 \mathrm{~kg}$ de grasa sobrepasante y $0,12 \mathrm{~kg}$ de una base mineral (Cuadro 1). Posteriormente, se ajustó el consumo de alimento balanceado conforme a la producción de leche en relación 3:1 (leche:alimento balanceado).

De las 40 vacas que consumieron esta dieta basal, 20 fueron suplementadas diaria e individualmente con 3,0 mg de Se obtenidos a partir de la Saccharomyces cerevisiae CNCM I-3060. Esta selenolevadura fue incorporada en $1,0 \mathrm{~kg}$ de alimento balanceado que fue suministrado previo al ordeño de la tarde a partir del día 5 hasta el día 56 post-parto. La concentración de Se en la dieta basal varió entre 0,67 y $0,72 \mathrm{mg} \cdot \mathrm{kg}^{-1}$ MS mientras que durante el período de suplementación, la concentración de Se en la dieta de las vacas suplementadas osciló entre 0,80 y 0,91 mg.kg-1 MS (Cuadro 2).

Cuadro 1. Composición nutricional de los ingredientes que componen la dieta basal de las vacas en el estudio.

\begin{tabular}{lccc}
\hline Ítem & RTM & Kikuyo & $\begin{array}{c}\text { Alimento Se } \\
\text { enriquecido }\end{array}$ \\
\hline Materia seca (\%) & 39,0 & 12,7 & 87,7 \\
Ingredientes (\% MS) & 37,1 & & \\
Alimento balanceado & 23,2 & & \\
Cebada & 21,5 & & \\
Silopaca de Kikuyo & 15,7 & & 18,7 \\
Melaza & 1,24 & & 18,6 \\
Grasa sobrepasante & 1,24 & & 1,86 \\
Base mineral & & & 3,49 \\
Nutrientes (Base MS) & 20,0 & 23,4 & 56,5 \\
PC $(\%)$ & 32,2 & 1,51 & 0,16 \\
FDN $(\%)$ & 1,68 & \\
EN $\left(\right.$ Mcal.kg-1) $_{\text {Se }\left(\mathrm{mg} . \mathrm{kg}^{-1}\right)}$ & 1,04 & & \\
\hline
\end{tabular}


Cuadro 2. Estimación del CMS, concentración y consumo de Se durante el período experimental.

\begin{tabular}{|c|c|c|c|c|c|c|c|c|c|c|}
\hline \multirow{2}{*}{ Ítem } & \multicolumn{5}{|c|}{ Control } & \multicolumn{5}{|c|}{ Experimental } \\
\hline & 5 & 14 & 28 & 42 & 56 & 5 & 14 & 28 & 42 & 56 \\
\hline CMS total, kg.vaca ${ }^{-1}$ & 16,4 & 20,5 & 20,3 & 19,8 & 21,1 & 15,3 & 20,7 & 20,0 & 19,3 & 20,6 \\
\hline \multicolumn{11}{|c|}{ Consumo de Se, mg.vaca ${ }^{-1}$} \\
\hline Absorbible $^{1}$ & 5,1 & 6,2 & 6,3 & 6,4 & 6,5 & 6,8 & 7,9 & 8,0 & 8,0 & 8,2 \\
\hline Total & 11,5 & 13,9 & 14,1 & 14,3 & 14,5 & 13,9 & 16,5 & 16,7 & 16,6 & 17,1 \\
\hline \multicolumn{11}{|c|}{ Concentración de Se, mg. $\mathrm{kg}^{-1} \mathrm{MS}$} \\
\hline Absorbible & 0,31 & 0,30 & 0,31 & 0,32 & 0,31 & 0,44 & 0,38 & 0,40 & 0,41 & 0,40 \\
\hline Total & 0,70 & 0,67 & 0,69 & 0,72 & 0,69 & 0,91 & 0,80 & 0,83 & 0,86 & 0,83 \\
\hline
\end{tabular}

${ }^{1}$ Se consideró una absorción de 45\% para el Se inorgánico y 66\% para la selenolevadura (Weiss 2005).

\section{Procedimientos de muestreo y mediciones}

\section{Estimación del consumo de materia seca y análisis en alimentos}

La disponibilidad del forraje se estimó mensualmente con la metodología denominada Botanal ${ }^{\circledR}$ descrita por Hargraves y Kerr (1978), mientras que el CMS proveniente del forraje se estimó por el método de reversa (van der Grinten et ál. 1992). La composición nutricional del forraje e ingredientes de la RTM fue determinada mensualmente por medio de muestras representativas a las que se les analizó el contenido de MS y PC según la metodología propuesta por AOAC (2005), de FDN conforme a la metodología de Van Soest y Robertson (1985) y la $\mathrm{EN}_{\mathrm{L}}$ fue estimada a través de las ecuaciones del NRC (2001).

\section{Producción y composición láctea}

En los días 5, 14, 28, 42 y 56 del período experimental, se midió individualmente la producción de leche y se tomaron muestras durante el ordeño de la mañana para determinar el contenido de grasa, proteína y lactosa (MilkoScan ${ }^{\mathrm{TM}}$ FT120, Foss Electric, Hillerød, DK), así como el CCS (Fossomatic ${ }^{\mathrm{TM}} 420$ Series, Foss Electric,
Hillerød, DK). Asimismo, se registró la incidencia de mastitis clínica.

\section{Análisis de selenio}

El contenido de Se en leche, forraje e ingredientes de la RTM fue determinado después de digerir $0,5 \mathrm{~g}$ de la muestra en una mezcla de 6 $\mathrm{ml}$ de ácido nítrico y $2 \mathrm{ml}$ de peróxido de hidrógeno al $30 \%$ mediante tubos de ensayo equipados con embudos de reflujo en un bloque de digestión calentado eléctricamente para reducir el Se (V) a Se (III). Posterior al proceso de digestión, los extractos resultantes fueron diluidos con agua bidestilada (10 $\mathrm{ml}$ volumen final) y analizados por espectrofotometría de absorción atómica (ZEEnit 700P, Analytik Jena AG, Jena, DE; Heard et ál. 2007). En todos los análisis se utilizó el material estándar de referencia SRM 1849a (Infant/Adult Nutritional Formula) certificado por el National Institute of Standards and Technology (NIST), con recuperaciones aceptables entre 81 y $107 \%$.

\section{Hematología y química sanguínea}

Durante el ordeño de la mañana de los días $5,14,28,42$ y 56 del período experimental, se obtuvieron muestras individuales de $4 \mathrm{ml}$ de sangre heparinizada mediante venopunción 
coccígea por medio del sistema de tubos al vacío para establecer el contenido de hemoglobina $(\mathrm{Hb})$ y la actividad en sangre de la enzima GSH-Px, como parámetros hematológicos y químicos, respectivamente, con un analizador químico clínico (Mindray BS-380, Shenzhen Mindray Bio-Medical Electronics Co., Ltd, Shenzhen, CN). Concretamente, para determinar la actividad en sangre de la GSH-Px a $37^{\circ} \mathrm{C}$, se mezclaron $0,05 \mathrm{ml}$ de sangre con $2 \mathrm{ml}$ de agente diluyente con el apoyo de un kit comercial (Ransel, Randox Laboratories Ltd. Antrim, UK) acorde con la metodología establecida por Paglia y Valentine (1967).

\section{Evaluación reproductiva}

Los ovarios de las vacas fueron examinados en los días 22 y 57 post-parto con ultrasonografía transrectal utilizando una sonda linear de $8,5 \mathrm{MHz}\left(\mathrm{Ibex}^{\mathrm{TM}}\right.$ Pro, E.I. Medical Imaging, Loveland, CO) para determinar el diámetro folicular. Adicionalmente, se registró el intervalo parto-primer servicio, intervalo partoconcepción, servicios por concepción, proporción de vacas servidas después de 90 días, así como la estimación de pérdidas económicas por incremento en los días abiertos.

\section{Análisis estadístico}

Los datos obtenidos fueron analizados mediante el procedimiento MIXED de SAS (SAS/STAT, SAS Institute Inc., Cary, NC; 2004), donde la vaca fue considerada como variable aleatoria mientras que el número de lactancia y el CCS inicial, fueron consideradas como covariables. A su vez, el efecto del tratamiento para las variables evaluadas se consideró como una tendencia cuando $0,05<\mathrm{p} \leq 0,10$ y significativo cuando $\mathrm{p} \leq 0,05$, en este caso se realizó la comparación entre medias por medio de la prueba de Waller-Duncan.

\section{RESULTADOS Y DISCUSIÓN}

\section{Producción y composición láctea}

La producción y composición láctea no difirieron entre tratamientos durante el período de suplementación (Cuadro 3). Estos resultados

Cuadro 3. Efecto de la suplementación post-parto con selenolevadura sobre la producción y composición láctea de vacas lecheras en pastoreo.

\begin{tabular}{|c|c|c|c|c|c|c|c|c|c|c|c|}
\hline \multirow{2}{*}{ Ítem } & \multicolumn{5}{|c|}{ Control } & \multicolumn{5}{|c|}{ Experimental } & \multirow{2}{*}{ EEM } \\
\hline & 5 & 14 & 28 & 42 & 56 & 5 & 14 & 28 & 42 & 56 & \\
\hline Leche $\mathrm{kg}$ & 25,9 & 35,5 & 39,6 & 40,7 & 41,7 & 23,8 & 35,9 & 38,8 & 39,6 & 40,2 & 1,30 \\
\hline \multicolumn{12}{|c|}{ Composición \% } \\
\hline Grasa & 4,28 & 3,67 & 3,30 & 3,16 & 3,04 & 4,09 & 3,46 & 3,24 & 2,97 & 3,06 & 0,20 \\
\hline Proteína & 3,90 & 3,38 & 2,89 & 2,80 & 2,81 & 3,98 & 3,31 & 2,91 & 2,81 & 2,74 & 0,06 \\
\hline Lactosa & 4,12 & 4,64 & 4,68 & 4,61 & 4,62 & 4,18 & 4,70 & 4,74 & 4,73 & 4,70 & 0,07 \\
\hline \multicolumn{12}{|c|}{ Componentes kg. $\mathrm{d}^{-1}$} \\
\hline Grasa & 1,19 & 1,25 & 1,28 & 1,27 & 1,23 & 1,06 & 1,19 & 1,29 & 1,17 & 1,27 & 0,09 \\
\hline Proteína & 1,01 & 1,20 & 1,17 & 1,14 & 1,18 & 1,01 & 1,19 & 1,14 & 1,13 & 1,14 & 0,04 \\
\hline Lactosa & 1,07 & 1,65 & 1,87 & 1,89 & 1,94 & 0,97 & 1,67 & 1,83 & 1,87 & 1,88 & 0,07 \\
\hline CCS $\log ^{1}$ & 5,30 & 4,10 & 2,75 & 2,75 & 2,97 & 4,88 & 2,98 & 2,37 & 2,42 & 1,76 & 0,74 \\
\hline
\end{tabular}

${ }^{1}$ Transformación logarítmica del CCS (y=Log 2 CCS/100) +3), según Dabdoud y Shook (1984).

EEM=Error Estándar de la Media. 
respaldan el hecho de que es poco probable que tanto la fuente como el contenido de Se en la dieta afecte la producción de leche o la concentración de sus componentes (Juniper et ál. 2006, Heard et ál. 2007, Walker et ál. 2010, Stockdale et ál. 2011).

Sin embargo, en estudios realizados en Australia y Nueva Zelanda, donde por lo general las pasturas suelen contener niveles marginales de Se $\left(<0,03 \mathrm{mg} \cdot \mathrm{kg}^{-1}\right)$ se han registrado respuestas en la producción de leche (Combs y Lu 2001). Asimismo, Tasker et ál. (1987) resaltan que podría esperarse una respuesta en la producción de leche en animales con concentraciones de Se en sangre inferiores a 9,5 $\mu \mathrm{g} . \mathrm{l}^{-1}$, pues según Silvestre et ál. (2007) un aumento en la actividad de la selenoproteína ID, la cual se encarga de regular procesos metabólicos, podría ocasionar incrementos de 2,0-2,5\% en la producción de leche.

Por su parte, a pesar de que Weiss et ál. (1990) establecieron una relación entre el incremento en la concentración plasmática de Se en vacas lactantes y la reducción en el CCS, el presente estudio no comprobó una reducción estadísticamente significativa del CCS, probablemente debido a que el CCS en el grupo denominado Control fue relativamente bajo.

Weiss (2003) y Silvestre et ál. (2007) sugieren que el beneficio de la suplementación con Se sobre la salud de la glándula mamaria se debe a los efectos sobre los neutrófilos y otras células inmunes. En este sentido, Velichko et ál. (2006) reportaron una disminución del 14,5\% en el CCS y una reducción del 54 y $89 \%$ en los casos de mastitis clínica y subclínica, respectivamente. En la presente investigación, no se presentaron casos de mastitis clínica en las vacas suplementadas con selenolevadura mientras que la incidencia en el grupo Control fue de $15 \%$.

\section{Contenido de selenio en leche}

La concentración promedio de Se en leche en las vacas previo a la suplementación (5 d) fue $10,6 \mu \mathrm{g} .1^{-1}$, dato afín a los valores reportados en la literatura para vacas lecheras. La suplementación con selenolevadura incrementó significativamente tanto la concentración como la excreción de Se en la leche durante el período experimental $(\mathrm{p}<0,01 ;$ Figura 1).

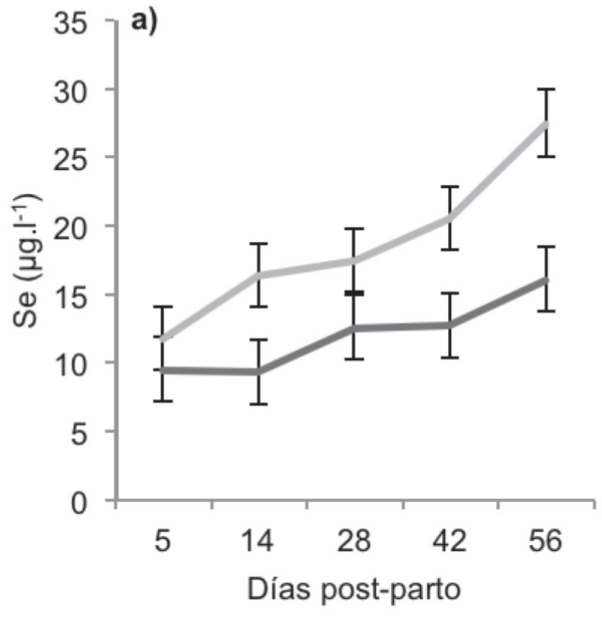

Control Experimental

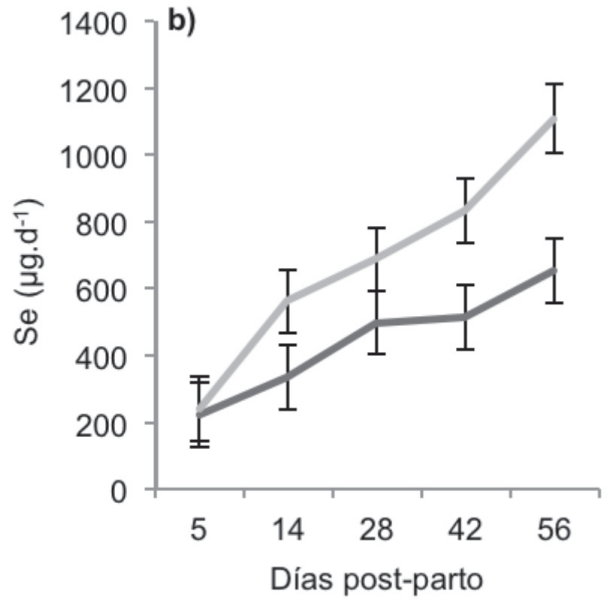

Control Experimental

Fig. 1. Efecto de la suplementación post-part con selenolevadura sobre a) la concentración y b) la excreción de Se en leche de vacas lecheras en pastoreo. 
De igual forma, la eficiencia aparente de la transferencia del Se a la leche, expresada como la cantidad de Se secretado en la leche en proporción al Se consumido, fue afectada por la suplementación con selenolevadura $(\mathrm{p}<0,01)$. En este sentido, en las vacas del grupo Control la eficiencia promedio fue $7,9 \%(5,4-10,1 \%)$ en comparación con 9,9\% (7,1-13,5\%) registrado en las vacas del grupo Experimental.

Sin embargo, esta proporción del Se consumido transferido a la leche, podría considerarse pequeña en comparación con el $17 \%$ reportado por Walker et ál. (2010) pero similar a los valores

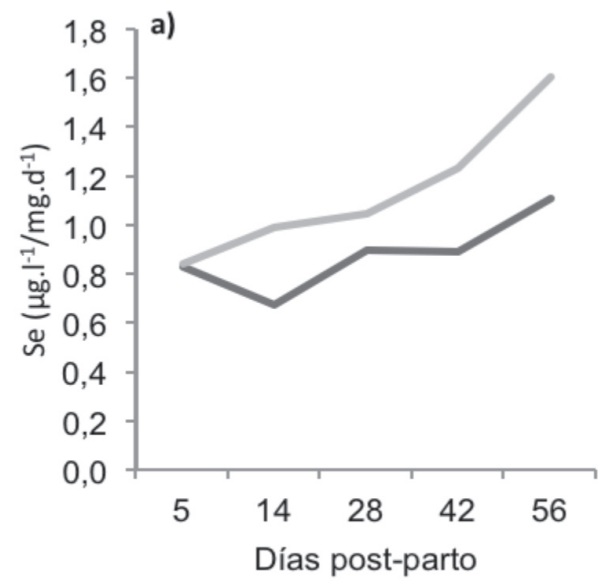

Control Experimental reportados por Heard et ál. (2007) en vacas en lactancia tardía (10-14\%); no obstante, los autores encontraron que estas vacas secretan una mayor proporción de Se en leche durante la etapa inicial de su lactancia (20-28\%).

La transferencia del Se dietético a la leche fue marcadamente más eficiente en las vacas suplementadas con selenolevadura (Figura 2), lo que reafirma que el Se proveniente de esta fuente es mejor absorbido que el Se derivado de formas inorgánicas, situación debida probablemente a la composición de aminoácidos de las proteínas de la leche (Weiss 2005).

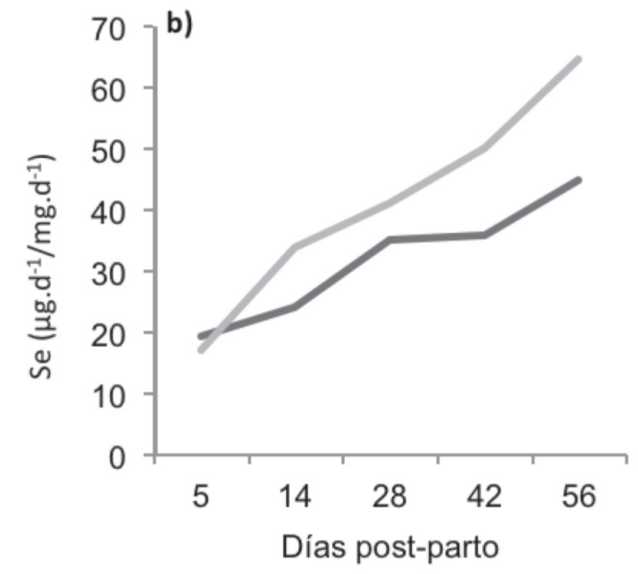

Control Experimental

Fig. 2. Efecto del incremento en el consumo total de Se post-parto sobre a) la concentración y b) la excreción de Se en leche de vacas lecheras en pastoreo.

El incremento relativo promedio en la concentración de Se en leche al suplementar con selenolevadura fue de $61 \%$ (20,5 vs. $\left.12,7 \mu \mathrm{g} . \mathrm{l}^{-1}\right)$, el cual se encuentra por debajo del $90 \%$ reportado en una revisión de 10 estudios realizada por Weiss (2005). Sin embargo, se identifica dentro del amplio rango existente en la respuesta a la suplementación con selenolevadura (Figura 3), ya que se han reportado incrementos relativos en la concentración de Se en leche desde un 15\% (Fisher et ál. 1995) hasta 160\% (Knowles et ál. 1999).
De acuerdo con Heard et ál. (2007), muchas compañías de lácteos han aumentando su interés en el desarrollo de productos especializados o enriquecidos, específicamente en relación con el Se y el desafío es desarrollar sistemas de alimentación en lecherías que produzcan leche con concentraciones de Se consistentes y predecibles para que los productos enriquecidos con Se puedan cumplir con las especificaciones predeterminadas, lo cual puede ser alcanzado a través de la inclusión del mineral en el alimento 


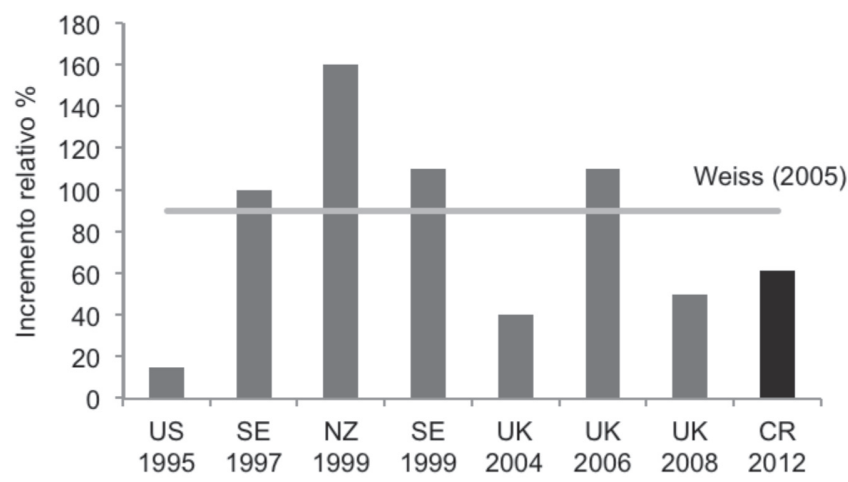

Fig. 3. Incremento relativo en la concentración de Se en leche de vacas lecheras suplementadas con selenolevadura respecto a una fuente inorgánica reportado en diferentes investigaciones.

balanceado o la premezcla mineral para obtener consumos consistentes dentro del hato.

Con respecto a lo anterior, Givens et ál. (2004) establecieron la relación entre el Se dietético y la concentración de Se en leche mediante regresiones lineales. En el presente estudio, la dispersión de los datos mostrada en la Figura 4a indica una inadecuada relación entre la concentración de Se en la dieta y la concentración de Se en la leche por lo que no podría considerarse tan buen predictor de la concentración de Se en leche como lo es el consumo de Se (Figura 4b), de manera que las regresiones lineales que establecen la relación entre el consumo de Se y la concentración de Se en leche fueron:

Control: Concentración de Se en leche $\left(\mu \mathrm{g} .1^{-1}\right)$

$=9,44 \times$ consumo Se total $\left(\mathrm{mg} \cdot \mathrm{d}^{-1}\right)-121,34 \quad \mathrm{R}^{2}=0,92$

Experimental: Concentración de Se en leche $\left(\mu \mathrm{g} .1^{-1}\right)$

$=18,19 \mathrm{x}$ consumo Se total $\left(\mathrm{mg} \cdot \mathrm{d}^{-1}\right)-283,46 \quad \mathrm{R}^{2}=0,82$

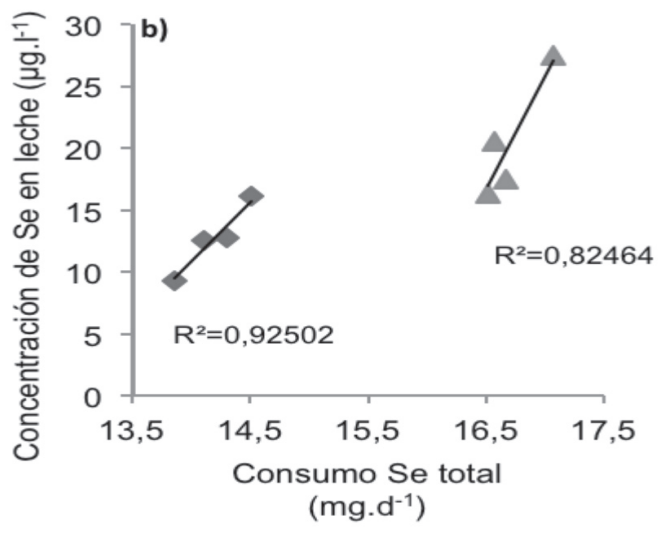

Control $\Delta$ Experimental

Fig. 4. Relación entre a) la concentración de Se en la dieta y b) el consumo de Se sobre la concentración de Se en leche de vacas lecheras en pastoreo. 
La respuesta en la concentración de Se en leche fue de $8,7 \mu \mathrm{g} . \mathrm{l}^{-1}$ por cada mg de selenolevadura consumido lo que se asemeja a lo encontrado por Heard et ál. (2007) y Walker et ál. (2010) quienes conjuntamente reportaron una respuesta de $7 \mu \mathrm{g} .1^{-1}$ por cada mg de Se consumido mientras que Stockdale et ál. (2011) reportaron $5 \mu \mathrm{g} . \mathrm{l}^{-1}$.

$\mathrm{El}$ incremento en la concentración de $\mathrm{Se}$ en leche encontrado en este experimento muestra que suplementar vacas lecheras con selenolevadura podría ser una vía útil para incrementar el consumo de Se en humanos. Actualmente, en Costa Rica no existen lineamientos que sugieran cual debería ser la concentración de Se en leche; sin embargo, Aspila (1991) propuso que una concentración deseable debería ser alrededor de $20 \mu \mathrm{g} . \mathrm{l}^{-1}$.

En el presente estudio, dicha concentración no fue alcanzada en el grupo Control (9,3-16,1 $\left.\mu \mathrm{g} .1^{-1}\right)$ mientras que en el grupo Experimental la concentración osciló entre 16,4 y $27,5 \mu \mathrm{g} .1^{-1}$ durante el período de suplementación. Aspila (1991) concluye que cuando se utiliza una fuente de Se inorgánica como selenito de sodio, las vacas deberían ser alimentadas con dietas que contengan alrededor de 0,7 mg.kg ${ }^{-1} \mathrm{MS}$, lo cual supera las máximas concentraciones permitidas, a pesar de que se asemejan a las concentraciones determinadas en el grupo Control.

La recomendación dietética diaria de Se para humanos depende del sexo y la edad, pero el requerimiento diario para adultos alcanza los 55 $\mu \mathrm{g}$ (Institute of Medicine 2000). Actualmente en Costa Rica, la concentración de Se en leche ronda los 5,4 $\mu \mathrm{g} . \mathrm{l}^{-1} \quad\left(3,2-8,0 \mu \mathrm{g} \cdot \mathrm{l}^{-1}\right.$; valores obtenidos mediante monitoreo en fincas lecheras ubicadas en la zona de Alajuela, Ciudad Quesada y Guanacaste, datos sin publicar).

Por lo que si se considera un consumo diario de $250 \mathrm{ml}$ de leche, el aporte de Se proveniente de la leche a la recomendación diaria actual sería de 2,6\%. Acorde con estos resultados, para proveer al menos un $10 \%$ de la recomendación dietética diaria de Se en $250 \mathrm{ml}$ de leche (22 $\left.\mu \mathrm{g} . \mathrm{l}^{-1}\right)$, las vacas deberían consumir diariamente al menos 2,2 $\mathrm{mg}$ de selenolevadura para ser un alimento fuente de Se.

\section{Actividad de la enzima Glutatión Peroxidasa}

La actividad de la enzima GSH-Px y la concentración de Se en sangre promedio en las vacas previo a la suplementación (5 d) fue 219,7 U.g ${ }^{-1} \mathrm{Hb}$ y $183,7 \mu \mathrm{g} . \mathrm{l}^{-1}$, respectivamente. La suplementación con selenolevadura no alteró significativamente la actividad de la GSH-Px ni la concentración de Se en sangre durante el período experimental, resultados similares a los reportados por Knowles et ál. (1999) en Nueva Zelanda y Juniper et ál. (2006) en el Reino Unido.

A pesar de esta situación, al suplementar con selenolevadura la actividad de la GSH-Px fue numéricamente mayor respecto al grupo Control, que presentó un incremento relativo promedio de $11 \%$ (220,3 vs. 199,0 ${\mathrm{U} . \mathrm{g}^{-1}}^{1}$ de Hb), ligeramente menor al $16 \%$ reportado en la revisión realizada por Weiss (2005). La principal respuesta en dichos estudios fue particularmente notoria cuando el consumo total de Se en el grupo Control fue relativamente bajo.

Tal es el caso de Knowles et ál. (1999) quienes encontraron respuesta en la GSH-Px cuando el consumo total de Se fue 2,0 mg.d ${ }^{-1}$ mas no cuando consumieron 4,0 mg.d $\mathrm{d}^{-1}$. Asimismo, los autores destacan que esta menor respuesta con respecto a la leche puede deberse a que ésta tiene una concentración de Met aproximadamente 2 veces mayor que la proteína de la sangre, por lo que es 2 veces más probable que la SeMet sea incorporada en las proteínas de la leche en lugar de las sanguíneas.

En una investigación de 12 semanas realizada en Suecia por Ortman y Pehrson (1999) se compararon los efectos de la fuente de Se, la actividad de la GSH-Px en vacas que consumieron selenato, selenito o SeMet, y se mantuvieron (los efectos) relativamente constantes durante las primeras 6 semanas del estudio, pero luego se incrementó marcadamente hasta la semana 12.

Estos resultados son evidencia de una fase de latencia en la actividad de la GSH-Px, la cual se incrementa rápidamente posterior a la 
incorporación del Se en la GSH-Px durante su nueva síntesis en la eritropoyesis (Knowles et ál. 1999). Es probable que en este estudio el período experimental de 7 semanas pudo haber limitado cambios en los eritrocitos y por ende las concentraciones de GSH-Px y de Se en sangre total, por lo que posibles resultados podrían verse después de 120-130 días de suplementación.
Debido a que en las vacas la concentración de Se en sangre es altamente correlacionada con la actividad de la GSH-Px (Erskine et ál. 1987), el incremento relativo también fue de $11 \%$ $\left(184,2\right.$ vs. $\left.166,4 \mu \mathrm{g} . \mathrm{l}^{-1}\right)$, igualmente por debajo del $20 \%$ reportado por Weiss (2005), pero dentro del rango reportado en diferentes investigaciones (Figura 5).

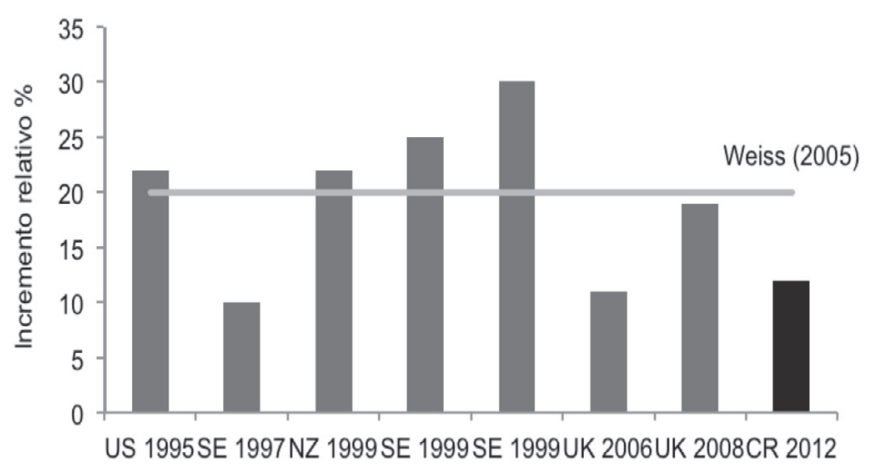

Fig. 5. Incremento relativo en la concentración de Se en sangre de vacas lecheras suplementadas con selenolevadura respecto a una fuente inorgánica reportado en diferentes investigaciones.

Se ha considerado que para alcanzar una óptima capacidad inmune y fertilidad, un nivel adecuado de GSH-Px debe ser de $130{\mathrm{U} . \mathrm{g}^{-1} \mathrm{Hb}}$ (Ceballos y Wittwer 1996). La proporción de vacas con un nivel de marginal de GSH-Px fue menor en el grupo Experimental respecto al Control (7,7 vs. $12,2 \%)$, ligeramente superior al $5,3 \%$ reportado por Ceballos et ál. (2003) en Colombia. Sin embargo, si se considera la concentración de Se en sangre como indicador, dicha proporción es mucho mayor puesto que el 61,5 y $63,4 \%$ de las vacas en el grupo Experimental y Control no alcanzaron los $180 \mu \mathrm{g} .1^{-1}$ recomendados por Weiss (2011).

\section{Parámetros de fertilidad}

Cerri et ál. (2009) han demostrado que tanto la deficiencia de Se como la presencia en exceso de especies reactivas de oxígeno (oxidantes) afectan negativamente la fertilidad, por lo que un adecuado accionar de la GSH-Px propiciaría un sistema antioxidante más eficiente para deshacerse de los radicales libres y generaría un impacto potencialmente positivo sobre la fertilidad y el desarrollo embrionario temprano.

Sin embargo, la incapacidad de la suplementación con Se orgánico mostrada en el presente estudio para alterar la actividad de la GSH-Px y la concentración de Se en sangre podría haber impedido algún efecto sobre parámetros de fertilidad (Cuadro 4), lo que coincide con lo encontrado por Rutigliano et ál. (2008) quienes reportaron que la fuente de Se no mejoró la actividad ovárica, la tasa de preñez ni la supervivencia embrionaria.

No obstante, a pesar de no encontrarse diferencias estadísticamente significativas, el diámetro folicular de las vacas suplementadas con selenolevadura fue numéricamente mayor que el de las vacas correspondientes al grupo Control, 
Cuadro 4. Efecto de la suplementación post-parto con Se sobre parámetros de fertilidad de vacas lecheras en pastoreo.

\begin{tabular}{lcc}
\hline Ítem & Control & Experimental \\
\hline Intervalo parto primer servicio, días & 99 & 85 \\
Intervalo parto concepción, días & 111 & 100 \\
Servicios por concepción, n & 1,29 & 1,27 \\
Primer servicio después de 90 d, \% & 50,0 & 36,4 \\
Diámetro folicular 22 d, mm & 12,8 & 13,0 \\
Diámetro folicular 57 d, mm & 13,1 & 16,0 \\
Pérdidas económicas, \$.vaca ${ }^{-1}$ & 56,0 & 48,1 \\
\hline
\end{tabular}

principalmente al día 57 post-parto. Al respecto, Mossa et ál. (2012) demostraron que las vacas con mayor tamaño folicular tenían 3 veces más posibilidades de preñarse al primer servicio en comparación con vacas que presentaban folículos de menor tamaño.

En este sentido, a pesar de no encontrarse diferencias significativas, el diámetro folicular de las vacas suplementadas con selenolevadura fue numéricamente mayor que el de las vacas del grupo Control, esta podría ser la razón por la cual en el presente estudio el intervalo parto concepción de las vacas suplementadas fue 11 días menor, lo que implicaría una retribución económica de $\$ 8$ por vaca.

\section{CONCLUSIONES}

La suplementación con selenolevadura no afectó significativamente la producción y composición láctea. Sin embargo, mejoró el nivel de Se en las vacas, lo que indica una mayor biodisponibilidad del Se proveniente de esta fuente.

La concentración de $\mathrm{Se}$ en leche en las vacas suplementadas con selenolevadura fue significativamente $(\mathrm{p}<0,05)$ mayor que en las vacas del grupo Control (61\%).

Incrementos en el nivel de inclusión de selenolevadura mostraron una efecto lineal positivo, que representa alrededor de $9 \mu \mathrm{g} . \mathrm{l}^{1}$ más por cada mg de Se consumido respecto al grupo Control.
La suplementación diaria con $3 \mathrm{mg}$ de $\mathrm{Se}$ en forma de selenolevadura permitiría alcanzar concentraciones de Se que admitan a la leche como un alimento fuente de este mineral y contribuir a reducir su deficiencia en la población costarricense.

La suplementación con selenolevadura no afectó significativamente los parámetros de fertilidad evaluados en este estudio.

\section{AGRADECIMIENTOS}

Los autores desean expresar su más sincero agradecimiento al señor Ricardo Gurdián Marchena, propietario de la finca donde se llevó cabo el ensayo. El agradecimiento es igualmente extensivo al médico veterinario Julio Murillo Barrantes por su colaboración en las evaluaciones reproductivas del experimento.

\section{LITERATURA CITADA}

AOAC (ASSOCIATION OF OFFICIAL AGRICULTURAL CHEMISTS). 2005. Official methods of analysis of AOAC. $18^{\text {th }}$ ed. AOAC. Arlington, VA. 2590 p.

ASPILA P. 1991. Metabolism of selenite, selenomethionine and feed-incorporated selenium in lactating goats and dairy cows. J. Agric. Sci. Finl. 63:1-74.

CEBAllOS A., CORREA H., LOAIZA J., VILLA N.A. 2003. Actividad sanguínea de glutatión peroxidasa como indicador del balance metabólico nutricional de selenio en rebaños lecheros de Manizales, Colombia. Rev. Col. Cienc. Pec. 16(1):19-25. 
CEBALlOS A., SÁNCHEZ J., STRYHN H. MONTGOMERY J.B., BARKEMA, H.W., WICHTEL J.J. 2009. Meta-analysis of the effect of oral selenium supplementation on milk selenium concentration in cattle. J. Dairy Sci. 92:324-342.

CEBAllos A., WITTWER F.G. 1996. Metabolismo del selenio en rumiantes. Archivos de Medicina Veterinaria 28(2):5-18.

CERRI R.L., RUTIGLIANO H.M., LIMA F.S., ARAÚJO D.B., SANTOS J.E. 2009. Effect of source of supplemental selenium on uterine health and embryo quality in high-producing dairy cows. Theriogenology 71:1127-1137.

CIGA (COMISIÓN INTERSECTORIAL DE GUÍAS ALIMENTARIAS). 2007. Actualización de lineamientos técnicos para la elaboración de las guías alimentarias de la población costarricense. $57 \mathrm{p}$.

COMBS G.F., LU L. 2001. Selenium as a cancer preventative agent, pp. 205-217. In: D. L. Hatfield (ed.). Selenium: Its molecular biology and role in human health Kluwer Academic Publishers. Norwell, MA.

DABDOUD S.M., SHOOK G.E. 1984. Phenotypic relations among milk yield, somatic cell count and clinical mastitis. J. Dairy Sci. 67:163-164.

DOYLE P.T., STOCKDALE C.R., JENKIN M.L., WALKER G.P., DUNSHEA F.R., SHIELDS P.M., MCKENNA A. 2011. Producing milk with uniform high selenium concentrations on commercial dairy farms. Anim. Prod. Sci. 51:87-94.

ERSKINE R.J., EBERHART R.J., HUTCHINSON J., SCHOLZ R.W. 1987. Blood selenium concentration and glutathione peroxidase activities in dairy herds with high and low somatic cell counts. JAVMA 11:1417-1421.

FISHER D.D., SAXTON S.W., ELLIOT R.D., BEATTY J.M. 1995. Effects of selenium source. Se status of lactating cows. Veterinary Clinical Nutrition 2(2):68-74

GIVENS D.I., ALLISON R., COTTRILL B., BLAKE J.S. 2004. Enhancing the selenium content of bovine milk through alteration of the form and concentration of selenium in the diet of the dairy cow. J. Sci. Food. Agric. 84:811-817.

HARGRAVES J.N.G., KERR J.D. 1978. Botanal: a comprehensive sampling and computing procedure for estimating pasture yield and composition. II. Computational package. Division of Tropical Crops and Pastures, CSIRO. Brisbane, Australia. 88 p.

HEARD J.W., STOCKDALE C.R., WALKER G.P., LEDDIN C.M., DUNSHEA F.R., MCINTOSH G.H., SHIELDS P.M., MCKENNA A., YOUNG G.P., DOYLE P.T. 2007. Increasing selenium concentration in milk: Effects of amount of selenium from yeast and cereal grain supplements. J. Dairy Sci. 90:4117-4127.
HIMENO S., IMURA N. 2000. New aspects of physiological and pharmacological roles of selenium. J. Health Sci. 46(6):393-398

INSTITUTE OF MEDICINE. 2000. Dietary reference intakes for vitamin $\mathrm{C}$, vitamin $\mathrm{E}$, selenium, and carotenoids. National Academy Press. Washington, DC. 529 p.

JUNIPER D.T., PHIPPS R.H., JONES A.K., BERTIN G. 2006. Selenium supplementation of lactating dairy cows: Effects of selenium concentration in blood, milk, urine, and feces. J. Dairy Sci. 89:3544-3551.

KNOWLES S.O., GRACE N.D., WURMS K., LEE J. 1999. Significance of amount and form of dietary selenium on blood, milk, and casein selenium concentrations in grazing cows. J. Dairy Sci. 82:429-437.

LYONS M.P., PAPAZYAN T.T., SURAI P.F. 2007. Selenium in food chain and animal nutrition: Lessons from nature. Asian-Aust. J. Anim. Sci. 20(7):1135-1155.

MINISTERIO DE SALUD. 2010. Encuesta nacional de nutrición 2008-2009. Micronutrientes. 2 p.

MOSSA F., WALSH S.W., BUTLER S.T., BERRY D.P., CARTER F., LONERGAN P., SMITH G.W., IRELAND J.J., EVANS A.C. 2012. Low numbers of ovarian follicles $\geq 3 \mathrm{~mm}$ in diameter are associated with low fertility in dairy cows. J. Dairy Sci. 95:2355-2361.

NRC (NATIONAL RESEARCH COUNCIL). 2001. Nutrient requirements of dairy cattle. $7^{\text {th }}$ revised edition. National Academy Press. Washington, DC. 408 p.

ORTMAN K., PEHRSON B. 1999. Effect of selenite as a feed supplement to dairy cows in comparison to selenite and selenium yeast. J. Anim. Sci. 77:33653370.

PAGLIA D.E., VALENTINE W.N. 1967. Studies on the quantitative and qualitative characterization of erythrocyte gluthatione peroxidase. J. Lab. Clin. Med. 70:158-169.

RUTIGLIANO H.M., LIMA F.S., CERRI R.L., GRECO L.F., VILELA J.M., MAGALHÃES V., SILVESTRE F.T., THATCHER W.W., SANTOS J.E.P. 2008. Effects of method of presynchronization and source of selenium on uterine health and reproduction in dairy cows. J. Dairy Sci. 91:3323-3336.

SAS INSTITUTE. 2004. SAS/STAT 9.1 User`s Guide. Version 9.1 ed. SAS Institute Inc., Cary, N.C. 5121 p.

SILVESTRE F.T., RUTIGLIANO H.M., THATCHER W.W., SANTOS J.E., STAPLES C.R. 2007. Effect of selenium source on production, reproduction, and immunity of lactating dairy cows. Florida Ruminant Nutrition Symposium. Gainesville, FL. 13 p.

STOCKDALE C.R., SHIELDS P.M., MCKENNA A., WALKER G.P., DUNSHEA F.R., DOYLE P.T. 2011. Selenium levels in cows fed pasture and concentrates or a total mixed ration and supplemented with selenized yeast to produce milk with supra- 
nutritional selenium concentrations. J. Dairy Sci. 94:262-272.

TASKER J.B., BEWICK T.D., CLARK R.G., FRASER A.J. 1987. Selenium response in dairy cattle. N.Z. Vet. J. 35:139-140.

VAN DER GRINTEN P., BAAYEN M.T., VILLALOBOS L., DWINGER R.H., MANNETJE L. 1992. Utilisation of kikuyu grass (Pennisetum clandestinum) pastures and dairy production in a high altitude region of Costa Rica. Tropical Grasslands 26:255-262.

VAN SOEST P.J., ROBERTSON J.B. 1985. Analysis of forages and fibrous feeds. Cornell University. Ithaca, NY. 165 p.

VELICHKO O.A., LOSEVA N.A., RYZHIY E.L., SADOVNIKOVA N.Y., SURAI P. 2006. Effect of long-term comsumption of Sel-Plex ${ }^{\circledR}$ and YeaSacc $₫ 1026$ on reproductive health of dairy cows. Alltech's 22nd Annual Symposium, (Abstract). Lexington, KY.

WALKER G.P., DUNSHEA F.R., HEARD J.W., STOCKDALE C.R., DOYLE P.T. 2010. Output of selenium in milk, urine, and feces is proportional to selenium intake in dairy cows fed a total mixed ration supplemented with selenium yeast. J. Dairy Sci. 93:4644-4650.

WEISS W.P. 2003. Selenium nutrition of dairy cows: comparing responses to organic and inorganic selenium forms, pp. 333-343. In: T.P. Lyons and K.A. Jacques eds. Nutritional Biotechnology in the Feed and Food Industries. Proceedings of Alltech's $19^{\text {th }}$ Annual Symposium. Nottingham University Press. Nottingham, UK.

WEISS W.P. 2005. Selenium sources for dairy cattle. Proc. Tri-State Dairy Nutrition Conference, pp 61-72. Ft. Wayne, IN.

WEISS W.P. 2011. Effects of micronutrients on the regulation of the immune system and its role in milk quality. $\mathrm{J}$. Dairy Sci. 94 (E-Suppl. 1):216.

WEISS W.P., TODHUNTER, D. A., HOGAN, J. S., SMITH, K. L. 1990. Effect of duration of supplementation of selenium and vitamin $\mathrm{E}$ on periparturient dairy cows. J. Dairy Sci. 73:3187-3194.

Todos los derechos reservados. Universidad de Costa Rica. Este artículo se encuentra licenciado con Creative Commons Reconocimiento-NoComercial-SinObraDerivada 3.0 Costa Rica. Para mayor información escribir a rac.cia@ucr.ac.cr 
